\title{
Valoración patrimonial de los yacimientos del Mioceno inferior del Barranco de Campisano de la Cuenca de Ribesalbes-Alcora (Araia d'Alcora, Castelló, España)
}

\author{
Mireia COSTA-PÉREZ ${ }^{*}$, Sergio ÁLVAREZ-PARRA², María Victoria PAREDES-ALIAGA3, \\ Óscar CABALLERO ${ }^{1}$, Esther BUENO ${ }^{1}$, Andreu VILAPLANA-CLIMENT ${ }^{1}$ \& Vicente D. \\ CRESPO $O^{4,5}$
}

${ }^{1}$ Departament de Botànica i Geologia, Universitat de València, c/ Doctor Moliner 50, 46100 Burjassot, Comunidad Valenciana, España.mireiacostap@gmail.com; oscachor@alumni.uv.es; estbue@alumni.uv.es; anvicli@alumni.uv.es

${ }^{2}$ Departament de Dinàmica de la Terra i de l'Oceà and Institut de Recerca de la Biodiversitat (IRBio), Facultat de Ciències de la Terra, Universitat de Barcelona, c/ Martí i Franquès s/n, 08028 Barcelona, España. sergio.alvarez-parra@ub.edu

${ }^{3}$ Instituto Cavanilles de Biodiversidad y Biología evolutiva, Universitat de València (UV), c/ Catedrático José Beltrán Martínez 2, 46980 Paterna, Comunidad Valenciana, España. mapa9@alumni.uv.es

${ }^{4}$ Museu Valencià d'Història Natural, c/ L'Hort de Feliu s/n, 46230 Alginet, Comunidad Valenciana, España. vidacres@gmail.com

${ }^{5}$ Museo Paleontológico de Alpuente, Av. San Blas 17, 46178 Alpuente, Comunidad Valenciana, España

* Corresponding author

Costa-Pérez, M., Álvarez-Parra, S., Paredes-Aliaga, M.V., Caballero, Ó., Bueno, E., Vilaplana-Climent, A. \& Crespo, V.D. 2019. Valoración patrimonial de los yacimientos del Mioceno inferior del Barranco de Campisano de la Cuenca de Ribesalbes-Alcora (Araia d'Alcora, Castelló, España). [Heritage value of the early Miocene localities of the Campisano ravine from RibesalbesAlcora Basin (Araia d'Alcora, Castellón, Spain)]. Spanish Journal of Palaeontology, 34 (1), 125-130.

Manuscript received 7 February 2019

Manuscript accepted 16 April 2019 https://doi 10.7203/sjp.34.1.15247

(C) Sociedad Española de Paleontología ISSN 2255-0550

\section{ABSTRACT}

In the present work the heritage valuation of the Spanish early Miocene localities of the Campisano ravine (RibesalbesAlcora Basin) is carried out. The results of the parameters according to the IELIG (Inventario Español de Lugares de Interés Geológico) methodology show the high value from the scientific and didactic points of view and the medium value from the tourist and recreational points of view. There is a medium risk of degradation, so it should be taken into account for short-term protection. Based on these data, the consideration of the localities as LIG (Lugar de Interés Geológico) and their inclusion in the IELIG are proposed.

Keywords: Palaeontological heritage, Ribesalbes-Alcora Basin, scientific value, LIG.

\section{RESUMEN}

En el presente trabajo se realiza una valoración patrimonial del conjunto de yacimientos del Mioceno inferior del barranco de Campisano (cuenca de Ribesalbes-Alcora). Los resultados obtenidos al calcular los parámetros según la metodología del Inventario Español de Lugares de Interés Geológico (IELIG) ponen de manifiesto el alto valor científico y didáctico de la zona, así como un valor turístico y recreativo medio. El riesgo de degradación es medio, por lo que debería ser tenido en cuenta para la protección de los yacimientos a corto plazo. En consonancia con estos datos, se propone la consideración de los yacimientos como LIG (Lugar de Interés Geológico) y su inclusión en el IELIG.

Palabras clave: Patrimonio paleontológico, cuenca de Ribesalbes-Alcora, valor científico, Lugar de Interés Geológico. 


\section{INTRODUCCIÓN}

La actividad minera en la cuenca de Ribesalbes-Alcora (Castelló, España) se ha centrado clásicamente en la explotación de las dolomicritas y lutitas laminadas bituminosas, además de las arcillas. Esta actividad se desarrolló desde el siglo XVIII hasta la actualidad. En el caso de las dos primeras, se explotaron hasta el primer cuarto del siglo $\mathrm{XX}$ principalmente en las minas de La Rinconada (Ribesalbes) y San Chils (Alcora), mientras que las arcillas se siguen explotando hasta la actualidad en la zona de Araia d'Alcora (Alcora) (Peñalver et al., 2016; Crespo, 2017). Esta cuenca se localiza al SE de la Cordillera Ibérica y consiste en un graben neógeno limitado por fallas normales que se extiende alrededor de $150 \mathrm{~km}^{2}$ (Fig. 1). Dos secuencias afloran en la cuenca: la secuencia de Alcora del Mioceno superior y la secuencia de Ribesalbes del Mioceno inferior. La segunda está dividida en cinco unidades de depósitos aluviales y lacustres, de las cuales las unidades $\mathrm{B}$ y $\mathrm{C}$ son ricas en contenido fosilífero
(Anadón et al., 1989). Los yacimientos de La Rinconada y San Chils pertenecen a la unidad B y han aportado un rico registro fósil de insectos, anfibios y plantas, además de coprolitos (Peñalver et al., 2016).

Los depósitos estudiados en este trabajo, situados en el barranco de Campisano, se hallan cerca del núcleo urbano de Araia d'Alcora. Estos depósitos pertenecen a la unidad C (Agustí et al., 1988), donde se han identificado 45 niveles fosilíferos en siete secciones (Fig. 1): Mas dels Coixos, Mas de Torner, Araia Cantera Sud, Barranc de Campisano, Foieta la Sarra, Mas d'Antolino B y Corral de Brisca. Se ha estudiado intensamente el registro fósil de mamíferos, dando como resultado una diversidad de 56 especies de mamíferos fósiles, además de otros vertebrados como cocodrilos o serpientes (Crespo, 2017; Crespo et al., 2019a). Los restos de mamíferos de estas secciones han facilitado su identificación con la biozona MN4 del Aragoniense inferior (Mioceno inferior) y su datación en unos 16,5-16 Ma (Crespo et al., 2019a). Entre los hallazgos más destacados se encuentra la descripción por primera vez

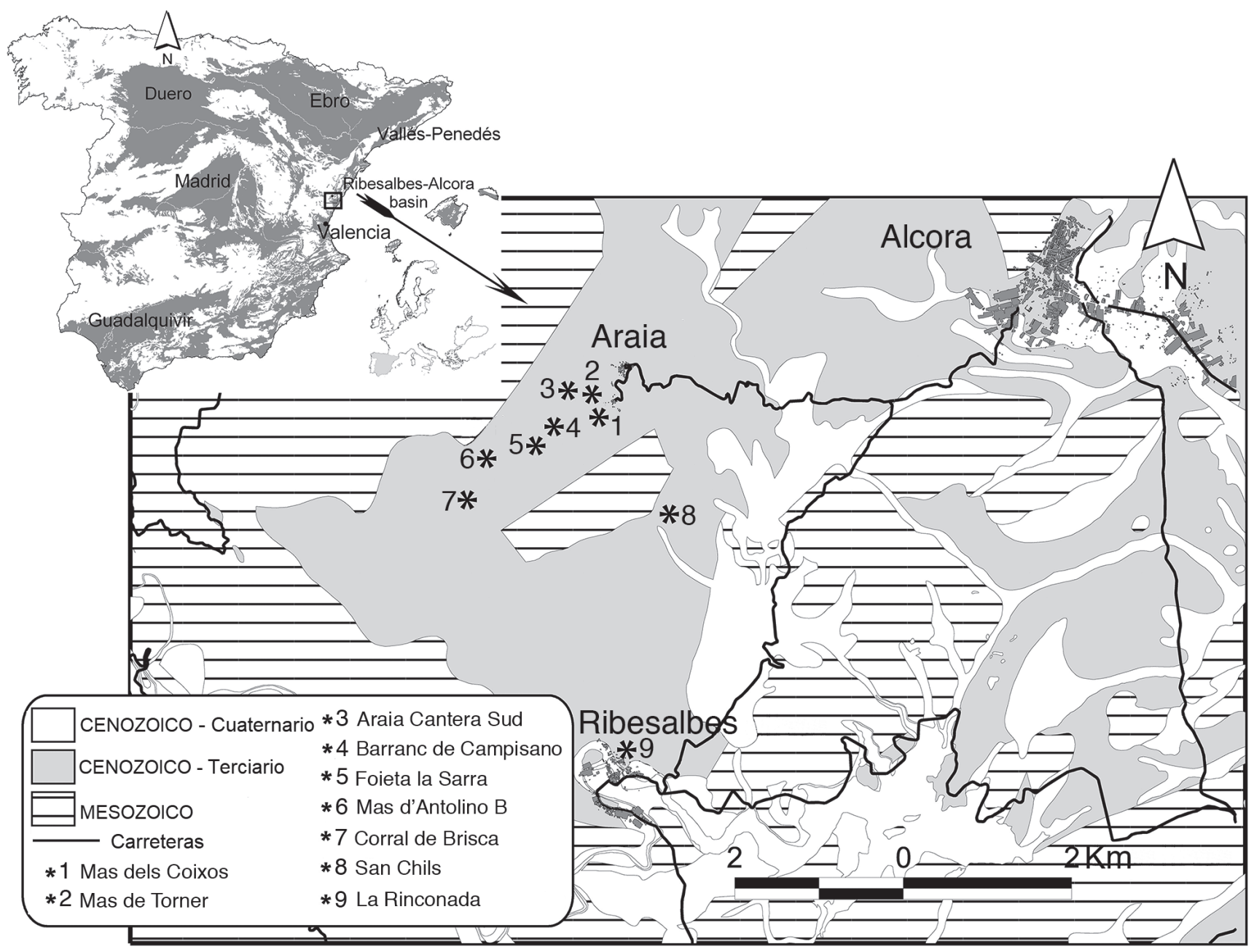

Figura 1. Localización geográfica y contexto geológico de la cuenca de Ribesalbes-Alcora, indicando las diferentes secciones en que se ha dividido el barranco de Campisano (1-7). Modificado de Crespo et al. (2019a). 
del dimílido Plesiodimylus ilercavonicus en la localidad tipo Mas d'Antolino B 5 (Crespo et al., 2018), así como en los yacimientos de Barranc de Campisano 1 y Mas d'Antolino B 11 (Crespo et al., 2019b), el registro más meridional del herpetotérido Amphiperatherium frequens (Furió et al., 2012) o la presencia de varias especies de tálpidos (Crespo et al., 2019b). Actualmente, el yacimiento de La Rinconada está incluido en el IELIG (Inventario Español de Lugares de Interés Geológico) con el código IB217 y se trata de la única área de la cuenca de Ribesalbes-Alcora que ha sido valorada patrimonialmente hasta el momento.

En este trabajo se realiza la valoración patrimonial de los yacimientos del Mioceno inferior del barranco de Campisano. Se ha utilizado la metodología propuesta por García-Cortés et al. (2014) con el objetivo de determinar su valor como posible LIG y, en función de ello, establecer en qué medida sería necesaria la conservación y protección de la zona.

\section{VALORACIÓN PATRIMONIAL PARA LA GESTIÓN Y PROTECCIÓN DE LOS YACIMIENTOS DEL MIOCENO INFERIOR}

La valoración patrimonial se ha realizado en conjunto para los yacimientos de las siete secciones del barranco de Campisano, ya que muestran similitudes en el registro fósil, las características tafonómicas, la litología y los factores de riesgo a los que están expuestos, además de situarse muy próximos entre sí. El yacimiento de La Rinconada posee un rico registro fósil, principalmente de plantas, insectos y anfibios, caracterizado por una preservación excepcional (Konservat-Lagerstätte) y una litología de rocas laminadas bituminosas. En cambio, el registro fósil de los yacimientos del barranco de Campisano incluye, principalmente, restos de vertebrados y gasterópodos y una litología que alterna margas, calizas y areniscas. Teniendo en cuenta estas diferencias, así como la distancia a la que se encuentran ambas áreas, se descartó la opción de incluir los yacimientos del barranco de Campisano en el LIG ya existente de La Rinconada. Por consiguiente, se ha realizado una valoración específica para estos yacimientos atendiendo a la propuesta metodológica de García-Cortés et al. (2014).

A partir del cálculo de los distintos parámetros, esta metodología permite asignar una puntuación para los valores científico, didáctico y turístico del lugar valorado (Tabla 1), los cuales pueden considerarse de forma independiente a la hora de seleccionar el lugar como LIG. En el estudio de los yacimientos del barranco de Campisano, el valor que ha resultado más destacable es el científico con una puntuación de 7 , por lo que se puede considerar como un LIG de muy alto valor (mayor de 6,65 sensu García-Cortés et al., 2014). Este alto valor vendría dado principalmente por el extenso trabajo de investigación realizado, por el cual se tiene un gran conocimiento científico del lugar, además de la presencia de abundantes restos de vertebrados y en especial de la localidad tipo de Plesiodimylus ilercavonicus (Crespo et al., 2018). Por otra parte, el uso didáctico de la zona representa un alto valor teniendo en cuenta su puntuación de 5 (entre 3,33 y $6,65)$. El hecho de ser un enclave paleontológico único a nivel regional le proporciona este valor didáctico, al igual que la diversidad de contenidos que puede ilustrar, tanto a nivel geológico como paleontológico. El uso turístico y recreativo resulta menos relevante, con una puntuación de 2,88 que indica un valor medio (inferior a 3,33). En este caso el potencial turístico de la zona es bajo debido principalmente a la escasez de infraestructuras logísticas y recreativas cercanas.

Tabla 1. Cálculo de los valores científico, didáctico y turísticorecreativo del LIG.

\begin{tabular}{|c|c|c|c|c|}
\hline Parámetros & Puntos & $\begin{array}{c}\text { Valor } \\
\text { científico }\end{array}$ & $\begin{array}{c}\text { Valor } \\
\text { didáctico }\end{array}$ & $\begin{array}{c}\text { Valor } \\
\text { turístico }\end{array}$ \\
\hline Representatividad & 2 & 60 & 10 & 0 \\
\hline Carácter de localidad tipo & 2 & 20 & 10 & 0 \\
\hline $\begin{array}{l}\text { Grado de conocimiento } \\
\text { científico del lugar }\end{array}$ & 4 & 60 & 0 & 0 \\
\hline Estado de conservación & 2 & 20 & 10 & 0 \\
\hline Condiciones de observación & 2 & 20 & 10 & 10 \\
\hline Rareza & 4 & 60 & 20 & 0 \\
\hline Diversidad & 4 & 40 & 40 & 0 \\
\hline Espectacularidad o belleza & 2 & 0 & 10 & 0 \\
\hline $\begin{array}{l}\text { Contenido didáctico/uso } \\
\text { didáctico detectado }\end{array}$ & 1 & 0 & 20 & 0 \\
\hline $\begin{array}{l}\text { Contenido divulgativo/uso } \\
\text { divulgativo }\end{array}$ & 2 & 0 & 0 & 30 \\
\hline Infraestructura logística & 2 & 0 & 30 & 10 \\
\hline Entorno socioeconómico & 1 & 0 & 0 & 10 \\
\hline $\begin{array}{l}\text { Asociación con elementos } \\
\text { del patrimonio }\end{array}$ & 4 & 0 & 20 & 20 \\
\hline Accesibilidad & 1 & 0 & 10 & 10 \\
\hline Densidad de población & 2 & 0 & 10 & 10 \\
\hline $\begin{array}{l}\text { Potencialidad para } \\
\text { actividades turísticas }\end{array}$ & 2 & 0 & 0 & 10 \\
\hline $\begin{array}{l}\text { Proximidad a zonas } \\
\text { recreativas }\end{array}$ & 1 & 0 & 0 & 5 \\
\hline Tamaño del LIG & 0 & 0 & 0 & 0 \\
\hline Total & & 7 & 5 & 2,88 \\
\hline
\end{tabular}

Una vez establecida la valoración que permita considerar los yacimientos como LIG se ha procedido a analizar en qué medida sería prioritaria su protección, para lo cual se 
deben aplicar otros parámetros que permiten valorar la susceptibilidad de degradación del lugar (Tabla 2). De este modo, y teniendo en cuenta factores tanto naturales como antrópicos, la susceptibilidad de degradación de la zona es media-alta en su conjunto. Aunque la susceptibilidad por amenazas naturales es media con 5 puntos (entre 3,33 y 6,66 sensu García-Cortés et al., 2014), cabe destacar la puntuación de 7 para las amenazas de carácter antrópico indicando una susceptibilidad alta (superior a 6,66), por lo que las posibilidades de alteración de la zona son elevadas. Aunque menos destacables, las amenazas naturales representan también riesgos a tener en cuenta, principalmente la erosión climática sobre la litología (margas blandas) y la bioerosión causada por la presencia de vegetación y la actividad de los animales. Por otro lado, las principales amenazas antrópicas corresponden a las actividades agrícolas y mineras de la zona, la construcción de viviendas, la acumulación de residuos en vertederos ilegales y la proximidad a actividades recreativas como el tránsito de motocicletas de montaña y otros vehículos. También es destacable la vulnerabilidad al expolio por el fácil acceso y la presencia de abundantes macrorrestos, como ya ha ocurrido en el yacimiento de La Rinconada.

Tabla 2. Valoración de la susceptibilidad de degradación antrópica $\left(\mathrm{S}_{\mathrm{DA}}\right)$ y degradación natural $\left(\mathrm{S}_{\mathrm{DN}}\right)$.

\begin{tabular}{lcc}
\hline \multicolumn{1}{c}{ Parámetros } & Puntos & Valor \\
\hline Interés por la explotación minera o hídrica & 2 & 50 \\
Vulnerabilidad de expolio & 4 & 100 \\
Proximidad a infraestructuras & 2 & 30 \\
Régimen de protección del lugar & 4 & 20 \\
Protección indirecta & 4 & 20 \\
Accesibilidad (agresión potencial) & 1 & 10 \\
Titularidad del suelo y acceso & 4 & 20 \\
Densidad de población & 2 & 10 \\
Cercanía a zonas recreativas & 4 & 20 \\
Total & & $\mathbf{2 8 0}$ \\
$\mathrm{S}_{\text {DA }}$ & & 7 \\
Factor tamaño del LIG (EF) & & $10 / 400$ \\
Fragilidad (F) & & 20 \\
Amenazas naturales (AN) & & 10 \\
$\mathrm{~S}_{\text {DN }}$ & & 5 \\
\hline
\end{tabular}

El valor de las amenazas de carácter antrópico se debe tener en cuenta en tanto que nos indica el riesgo de degradación del lugar, el cual mide el daño potencial y es indicativo de la necesidad o prioridad de protección que se debe aplicar. En este caso el valor obtenido es de 4,9 (Tabla 3), de tal forma que el riesgo de degradación sería medio (entre 3,33 y 6,66 ) y por lo cual el lugar debería ser objeto de medidas de protección a corto plazo (GarcíaCortés et al., 2014).

Tabla 3. Riesgo de degradación $\left(\mathrm{R}_{\mathrm{D}}\right)$.

\begin{tabular}{lc}
\hline \multicolumn{1}{c}{ Riesgo de degradación $\left(\mathbf{R}_{\mathrm{D}}\right)$} & Valor \\
\hline $\begin{array}{l}\text { Riesgo de degradación del valor científico por amenazas } \\
\text { naturales }\end{array}$ & 3,5 \\
$\begin{array}{l}\text { Riesgo de degradación del valor didáctico por amenazas } \\
\text { naturales }\end{array}$ & 2,5 \\
$\begin{array}{l}\text { Riesgo de degradación del valor turístico por amenazas } \\
\text { naturales }\end{array}$ & 1,44 \\
$\begin{array}{l}\text { Riesgo de degradación del LIG por amenazas naturales } \\
\left(\mathrm{R}_{\mathrm{DN}}\right)\end{array}$ & 3,5 \\
$\begin{array}{l}\text { Riesgo de degradación del valor científico por amenazas } \\
\text { antrópicas }\end{array}$ & 4,9 \\
$\begin{array}{l}\text { Riesgo de degradación del valor didáctico por amenazas } \\
\text { antrópicas }\end{array}$ & 3,5 \\
$\begin{array}{l}\text { Riesgo de degradación del valor turístico por amenazas } \\
\text { antrópicas }\end{array}$ & 2,01 \\
$\begin{array}{l}\text { Riesgo de degradación del LIG por amenazas antrópicas } \\
\left(\mathrm{R}_{\mathrm{DA}}\right)\end{array}$ & 4,9 \\
$\begin{array}{l}\text { Riesgo de degradación del valor científico } \\
\text { Riesgo de degradación del valor didáctico }\end{array}$ & 4,2 \\
$\begin{array}{l}\text { Riesgo de degradación del valor turístico } \\
\text { Riesgo de degradación del LIG ( } \mathrm{R}_{\mathrm{D}} \text { ) }\end{array}$ & 3 \\
\hline
\end{tabular}

\section{CONCLUSIONES}

El conjunto de yacimientos del Mioceno inferior del barranco de Campisano aporta información paleontológica que contribuye al conocimiento del registro fósil de mamíferos, como se puede observar en los múltiples estudios realizados sobre la zona (ver referencias en Crespo et al., 2019a). Es por ello que se ha aplicado la metodología del IELIG (García-Cortés et al., 2014) para realizar una valoración patrimonial del conjunto, obteniendo unos datos positivos en cuanto a su valor como posible LIG. Teniendo en cuenta estos resultados se puede afirmar que el lugar presenta un valor científico muy alto, motivo por el cual debería ser un candidato a considerar para su inclusión en el catálogo del IELIG. Puesto que el valor didáctico es también alto, se propone su uso en actividades divulgativas y/o académicas relacionadas con la paleontología, la geología y el medio ambiente, así como para dar a conocer el patrimonio paleontológico de la región. Por otra parte, cabe tener en cuenta la alta susceptibilidad a amenazas antrópicas, que da lugar a un riesgo medio de degradación o daño potencial sobre los yacimientos. Por estos motivos sería aconsejable aplicar medidas de protección a corto plazo para prevenir la posible degradación de estos yacimientos. Considerando 
que para una buena gestión de la zona debería estudiarse el caso en detalle, se proponen las siguientes medidas de protección: supervisión paleontológica de las actuaciones mineras y de construcción en la zona, control y retirada de los vertederos ilegales, prohibición de extracción de tierra procedente de las secciones para uso agrícola, impedir el paso de vehículos de recreo e implementación de medidas de disuasión para evitar el expolio.

\section{AGRADECIMIENTOS}

Queremos agradecer al Dr. Enrique Peñalver (IGME) por animarnos a llevar a cabo este trabajo, además de sus interesantes aportes sobre la cuenca de Ribesalbes-Alcora durante el trabajo de campo. Agradecemos también a los revisores Esperanza Fernández y Luís Carcavilla sus comentarios y sugerencias sobre el manuscrito original.

\section{REFERENCIAS}

Agustí, J., Anadón, P., Ginsburg, L., Mein, P. \& Moissenet, E. 1988. Araya et Mira: nouveaux gisements de mammifères dans le Miocène Infèrieur-Moyen des Chaînes Ibériques orientales et méditerranéennes. Conséquences stratigraphiques et structurales. Paleontologia i evolució, 22, 83-101.

Anadón, P., Cabrera, L., Julià, R., Roca, E. \& Rosell, L. 1989. Lacustrine oil-shale basins in tertiary grabens from NE Spain (Western European rift system). Palaeogeography, Palaeoclimatology, Palaeoecology, 70, 7-28; doi: 10.1016/0031-0182(89)90077-1.
Crespo, V.D. 2017. Los mamiferos del Mioceno inferior de la Cuenca de Ribesalbes-Alcora (Castelló, España). Ph.D. Tesis (inédita). Universitat de València, 695 pp.

Crespo, V.D., Furió, M., Ruiz-Sánchez, F.J. \& Montoya, P. 2018. A new species of Plesiodimylus (Dimylidae, Eulipotyphla, Mammalia) from the Early Miocene of Spain. Historical Biology, 30, 360-371; doi: 10.1080/08912963.2017.1289519.

Crespo, V.D., Marquina-Blasco, R., Ruiz-Sánchez, F.J. \& Montoya, P. 2019a. An unusual insectivore assemblage from the early Miocene of southwestern Europe: The talpids and dimylids from the Ribesalbes-Alcora Basin (Spain). Comptes Rendus Palevol, en prensa; doi. org/10.1016/j.crpv.2019.03.003.

Crespo, V.D., Suárez-Hernando, O., Murelaga, X., RuizSánchez, F.J. \& Montoya, P. 2019b. Early Miocene mammal assemblages from the Campisano ravine in the Ribesalbes-Alcora Basin (E Spain). Journal of Iberian Geology, 1-14; doi: 10.1007/s41513-018-0093-z.

Furió, M., Ruiz-Sánchez, F. J., Crespo, V.D., Freudenthal, M. \& Montoya, P. 2012. The southernmost Miocene occurrence of the last European herpetothetiid Amphiperatherium frequens (Metatheria, Mammalia). Comptes Rendus Palevol, 11, 371-377; doi:10.1016/j.crpv.2012.01.004.

García-Cortés, A., Carcavilla, L., Díaz-Martínez, E. \& Vegas, J. 2014. Documento Metodológico para la Elaboración del Inventario Español de Lugares de Interés Geológico $(L I G)$. Instituto Geológico y Minero de España, Ministerio de Economía y Competitividad, Versión 5/12/2014, 64 pp.

Peñalver, E., Barrón, E., Postigo Mijarra, J.M., García Vives, J.A. \& Saura Vilar, M. 2016. El Paleolago de Ribesalbes. Un Ecosistema de Hace 19 Millones de Años. Servicio de Publicaciones, Diputación de Castelló \& Instituto Geológico y Minero de España Eds., España, 201 pp. 
